\title{
Publisher Correction: Greener and cheaper
}

Laifa Shen and Yan Yu

Correction to: Nature Energy https://doi.org/10.1038/s41560-017-0026-7 (2017); published online 30 October 2017

In the HTML version of this News \& Views originally published, the corresponding author was incorrectly flagged as being Laifa Shen; it should have been Yan Yu. This has now been corrected. 